Original Research Paper

\title{
Semi-Probabilistic Calibration of a Partial Material Safety Factor for Structural Silicone Adhesives - Part I: Derivation
}

\author{
${ }^{1}$ Michael Drass and ${ }^{2}$ Michael A. Kraus \\ ${ }^{1}$ Department of Civil and Environmental Engineering, Institute of Structural Mechanics and Design, Germany \\ ${ }^{2}$ Department of Civil and Environmental Engineering, Stanford University, USA
}

\author{
Article history \\ Received:11-12-2019 \\ Revised: 31-12-2019 \\ Accepted: 04-02-2020 \\ Corresponding Author \\ Michael Drass \\ Department of Civil and \\ Environmental Engineering, \\ Institute of Structural \\ Mechancis and Design, \\ Germany \\ E-mail: drass@ismd.tu-darmstadt.de
}

\begin{abstract}
This paper deals with the application of the semi-probabilistic design concept (level I) of Eurocode 0 in order to calibrate partial safety factors for structural silicone sealants. In the first part of this article the current legal situation for the application of structural sealants in façades is described, where a new Eurocode-conform design concept is introduced and compared to existing design codes (ETAG 002) or national design protocols (DIBt concept). Basic background on semiprobabilistic modelling and the general framework for deriving partial material safety factors at a level I stage for structural sealants is given then. The main part of this publication is concerned with determining the specific partial material safety factors for DOWSIL 993 silicone using existing experimental data, which were obtained under the ETAG 002 testing protocol. It is found, that the correct level I calibration of that partial material safety factors are significantly lower compared to currently existing estimates and thus allow for a great optimization of structural sealant design situation with potentially high economical as well as sustainability benefits.
\end{abstract}

Keywords: Partial Material Safety Factor, Structural Silicone Adhesive, SSG Façades, Design and Computation

\section{Introduction and Current Situation}

Modern glass façades are designed with a strong emphasis on a transparent appearance with minimal visibility of the supporting structures. In the last fifty years, a lot of experience has been gained worldwide with structural silicone adhesive joints in façade design. Starting with linear adhesive joints, which are used along a window system to ensure homogeneous load transfer (Staudt et al., 2018), up to local fixations, where glass panes are only bonded locally with so-called point fixings (Drass et al., 2019; Santarsiero and Louter, 2019). Recent developments deal with so-called laminated joints, where either a puck is laminated into a Laminated Safety Glass (LSG) or something is hoof laminated onto a glass (Bedon and Santarsiero, 2018).

There are two standards for dimensioning silicone adhesive joints in façades ETAG 002 (2012); ASTM C1401 (2002), which are common in practice all over the world. Both design methods are based on a linear analysis of the geometric and the material behaviour and assume an even load distribution. Further, a constant stress state is assumed for the adhesive, resulting in a nominal stress analysis. In this context, the nominal stress is the support reaction force divided by the reference nominal sealant area, resulting in a very simple manual proof of calculation, often sarcastically referred to as the 'Mickey Mouse' formula. These two standards use a global safety concept to provide sufficient redundancy or safety in the design of the silicone adhesive joint so that modelling inaccuracies (load and constitutive behaviour), temperature, humidity and ageing effects (salt, detergents, $\mathrm{SO}_{2}, \mathrm{UV}$ ) are covered. A global safety factor $\gamma_{\text {tot }}$ is therefore introduced, which means that the experimentally determined $5 \%$ quantile of the nominal tensile or shear strength is divided by this global safety factor of $\gamma_{\text {tot }}=6$ in order to achieve a sufficiently large safety margin (ETAG 002, 2012). This procedure yields so-called nominal design stresses for a tensile or shear loading, which have been calibrated on the ETAG $\mathrm{H}$-specimens. Unfortunately, the exact history of determining the global safety factor of $\gamma_{\text {tot }}=6$ cannot be reconstructed at this time based on the current version 
of ETAG 002 (2012). Therefore, a discussion about the safety factor has been sparked in the industry today and the demand for a comprehensible calculation of a correct and justifiable safety factor has arisen.

The use of structural sealants in Germany (and similarly in other European countries) has so far only been possible within the strict framework of an approval in individual cases or an European Technical Approval (ETA) for structural sealant façades. The requirements for these approvals were based on ETAG 002 (2012). A more recent but almost unknown concept has been proposed in Germany by the 'Deutsches Institut für Bautechnik' (DIBt Concept, 2012), in which the computation of a partial safety factor according to Eurocode 0 or EC 0 DIN EN 1990 (2010) is intended and allowed. The disadvantage of the DIBt concept lies in the fact that it is not publicly accessible, that it only applies locally to Germany and that the verification format or the determination of a partial safety factor is not fully defined.

In contrast to the conservative normative approaches, there are also ideas from research and development to present a design format for siliconebonded components, which is either a rough computation approach with misuse of mechanically or statistically predefined terms (Maniatis et al., 2015; Maniatis and Siebert, 2016) or which deal with a precise material and failure modelling (Santarsiero, 2015; Drass, 2019). Although all these approaches calculate improved global or partial safety factors, none of them offers a direct link to DIN EN 1990 (2010), which is based on a semi-probabilistic partial safety concept.

Since little work is currently being done on the methodically correct and thus Eurocode-compliant derivation of a partial safety factor for silicone adhesives in façade applications, this article deals on the one hand with the development and presentation of a Eurocode-compliant partial safety factor and on the other hand with the implementation of a Level I approximation of a partial safety factor for the silicone adhesive DOWSIL 993. However, the methodology is generally valid, so that a partial safety factor can also be derived for other structural silicones. The proposed procedure is based upon the calibration procedure given in Eurocode EC 0 and additionally uses test results and modelling contents from ETAG 002 (2012) to create a simple link between the two concepts. With the help of the determined partial safety factor, which was calibrated on the basis of test data provided by Seele Group $\mathrm{GmbH}$ and Co. KG, it is possible to design and compute silicone adhesive joints according to the partial safety concept of DIN EN 1990 (2010). The authors are anticipating the following results for the industry: On the one hand, a partial safety factor based on experimental data according to ETAG 002 (2012) is to be derived which replaces the global safety factor $\gamma_{\text {tot }}$ $=6$ of ETAG 002 (2012) since a consistent (but level I approximation) derivation according to EC 0 is now available. On the other hand, the structural verification of adhesive joints in façade applications is to be transferred to a partial safety concept according to DIN EN 1990 (2010), so that it is now possible to carry out the verification according to the currently valid standard. Furthermore, the discussion and bargaining about the existing global safety factor is to be silenced, since a mathematically founded and Eurocode-compliant derivation of the safety factor is now available.

This paper is organized as follows: Firstly, it is concerned with describing the state of the art of designing with structural sealants accordingly to currently available codes and procedures as well as latest research. Subsequently, a methodical protocol for the calibration of a partial material safety factor for structural silicone adhesives is proposed which is based on the procedures of DIN EN 1990 (2010). The proposed method is evaluated for the structural silicone adhesive DOWSIL 993 and a partial material safety factor with associated characteristic strengths is derived. The derivation of the partial safety factor in this paper refers only to ETAG > 002-compliant adhesive joints, so that the partial material safety factor is also deduced on the basis of ETAG 002 (2012). A conclusion highlights the findings of this paper and outlines further research topics w.r.t. more advance reliability assessment methods. Furthermore, the outline discusses the application of the presented concept for the determination of a partial material safety factor on adhesive joints which do not correspond to the geometrical restrictions of ETAG 002 (2012).

\section{State of the Art Normative Concepts for Structural Sealant Glazing Systems}

In order to better understand the current normative background on structural sealant glazing systems for the area of application of façade construction and also to understand the background of the design concepts, this section briefly summarises the concept according to ETAG 002 (2012) and the DIBt Concept (2012) and examines their limitations.

\section{Concept of ETAG 002}

ETAG 002 describes the calculation, testing and application of structural sealants in façades and roofs with a maximal inclination of $7^{\circ}$ above horizontal. It covers the adhesion between glass, either uncoated or with an inorganic coating and anodised aluminium or stainless steel. Specific requirements from ETAG 002 (2012) for supported (types I and II) and unsupported (types III and IV) systems can be summarized by: 
- Adhesive joint must be silicone in a linear bead

- Discontinuities in the structural bond are allowed, however no edge may be entirely free, except if it is mechanically fastened

- Adhesive joint must by applied in the factory

- More than two adhesion surfaces are not allowed

- Linear adhesive bead must have a minimal thickness of $e=6 \mathrm{~mm}$, whereas the lateral dimensions must lie within $6 \leq h_{c} \leq 20 \mathrm{~mm}$

To design with structural sealants in façade application, ETAG 002 (2012) proposes two methods. The first method uses design formulas, from which the dimensions of the adhesive joint are reversely calculated depending of the design stress (engineering stress measure) in the adhesive joint. The second method intends the dimensioning of adhesive joints based on simulations and research results, which at first sight sounds very vague, but also offers possibilities to deviate from the tough restrictions of ETAG 002 (2012).

Looking at the design stresses, the tensile yield design stress $\sigma_{\text {des, }}$, shear yield design stress under dynamic load $\Gamma_{\text {des }}$ and shear yield design stress under permanent load $\Gamma_{\infty}$ will be discussed in the following. The design stresses are determined by evaluating the $5 \%$ quantile yield (failure) stress measured on ETAG H-samples for tension and shear and dividing it by a global safety factor of $\gamma_{\text {tot }}=6$ if the adhesive is not permanently exposed to load. Thus, the design resistances are calculated by:

$$
\sigma_{\text {des }}=\frac{R_{u, 5}}{\gamma_{t o t}} \text { fortension and } \Gamma_{\text {des }}=\frac{R_{u, 5}}{\gamma_{t o t}} \text { for shear. }
$$

In this case, all data refer to the reference temperature of $23^{\circ} \mathrm{C}$ at which the ETAG $\mathrm{H}$-samples are tested. Although the global safety factor can be regulated nationally, the value of six is proposed to take account of influences from temperature, stress duration and inaccurate calculation methods. However, an exact derivation of the global safety factor is not documented, so that one can only vaguely assume which influences have been taken into account. In Germany, therefore, this value is often reduced to the value of $\gamma_{\text {tot }}=4$ as a result of more precise numerical computations of the adhesive joints, but it would in principle be appropriate to use a semi-probabilistic safety concept instead of a global safety factor in order to obtain a proper material safety factor corresponding to the method given in DIN EN 1990 (2010). Returning to the calculation of the design resistance for structures under constant load (dead load) of a glass pane, it is determined as follows:

$\Gamma_{\infty}=\frac{\Gamma_{\text {des }}}{\gamma_{c}}$ with $\gamma_{c}=10$.
From this it becomes clear, that the $5 \%$ quantile value of the experimentally determined shear strength is reduced by approximately $6000 \%$, since the long-term behaviour under constant load has not yet been substantially researched.

\section{DIBt Concept}

In Germany, for example, it is necessary to verify the load-bearing capacity by means of an increased experimental and/or numerical effort in accordance with the DIBt Concept (2012), if there is a slight deviation from the required geometric conditions according to ETAG. The expression of a slight geometric deviation is not clearly defined and is therefore always in need of discussion. However, there is a consensus that three-flank bonding or flat bonded joints cannot be verified with the mentioned concepts. This concept provides a five-point program to design and verify adhesive joints with slightly modified geometry (compared to ETAG 002). These points are briefly presented and summarized in the following:

\section{Step 1 Description of the Matter of Subject to} Authorisation

Data have to be provided by the applicant concerning design, choice of materials and intended field of application. This includes in particular the description of application area, climatic zones, installation situations including assembly system, measures for installation monitoring, component drawings with material specification, material data and constitutive laws for FE computations, actions onto the adhesive for the relevant stress states (compression, tension, shear, combination of tension-shear-compression) and the definition of a target service life period to be verified for a required safety level (usually 25 years)

\section{Step 2 Quantification of External Influences on the} Adhesive Joint

In particular, this involves the quantification of controlled impacts on a component with regards to its dead weight, snow and wind loads, additional damaging impacts such as physicalchemical impacts (UV, moisture, cleaning agents, erosols and trace gases as well as particle migration from materials in contact), determination of the mechanical loads and timerelated classification of the impacts according to quasi-static and periodically recurring stresses

\section{Step 3 Determination of the Local Extreme Stresses in the Adhesive Joint}

In this step, the aim is to simulate component tests carried out using validated mechanical-numerical models with the result of identifying so-called hot 
spots, which form the basis for experimental long-term and or fatigue validation with laboratory samples

\section{Step 4 Conduction of Long-Term and Fatigue Tests}

Based on the local stresses in the adhesive as determined in step 3, load values for adhesion and stress as well as creep and fatigue tests for example, are determined, on the basis of which a large test program with small-scale specimens must be carried out. There the geometry of the adhesive joint corresponds to the dimensions of its actual application

\section{Step 5 Assessment of the Safety Level of the Bonded Joint}

The last step is to describe and fulfil a verification concept regarding the static long-term strength, the verification under creep as well as dynamic tensilethreshold loading. On the basis of the conducted experiments, an equivalent stress for a service life cycle of 25 years is to be defined. Furthermore, partial safety factors must be computed for the sealant joint

Unfortunately, the derivation of the partial material safety factors according to DIBt Concept (2012) has never been carried out and the documentation provides only vague insights into how the safety factor value can be determined. It is therefore necessary to derive the partial material safety factor for silicone adhesives in accordance with the Eurocode 0.

\section{Calibration of a Partial Material Safety Factor for Structural Silicone Adhesives}

In this section, the general methodology for deriving a partial material safety factor according to DIN EN 1990 (2010) is presented and calibrated using the example of the structural adhesive DOWSIL 993.

\section{Semi-Probabilistic Safety Concept}

The safety and reliability of buildings is on the one hand determined by the variability within the actions and resistances of it and on the other hand by potential errors in planning, execution and use. Human misconduct however, cannot be detected, handled and covered by a safety concept, but must be excluded as far as possible by targeted measures such as checking of a structural design computation, quality assurance during the construction process of the structure and maintenance during use. Only the stochastic character of the input variables for actions and resistances can be determined by probabilistic methods. This requires a quantification of the stochastic uncertainties in actions and resistances.

Basically, the core of the design philosophy in DIN EN 1990 (2010) is the solution of the inequality:
$E_{d} \leq R_{d}$,

where, $E_{d}$ represents the design value of an action and $R_{d}$ the design value of the resistance.

In civil engineering, reliability is assessed by comparing the calculated reliability index with that one, considered sufficient for the system under assessment, from previous experience. For this purpose, one must establish a relationship between the capacity $R_{d}$ (for example, the strength) of the system and the demand $E_{d}$ (for example, the load) such that if capacity and demand are equal, there is a limiting state of interest. The margin of safety, defined as $S=g(E, R)=R-E$, is another example of this state, where $S>0$ represents the safe state, $S<0$ the failure state. For reasons of completeness, $S=0$ defines the limiting state. Accordingly, the probability of failure $p_{f}$ is given by:

$p_{f}=\operatorname{Pr}[R-E \leq 0]=\operatorname{Pr}[S \leq 0]$,

where the $\operatorname{Pr}[\bullet]$ defines any probability operator applied to the argument $\bullet$.

As an example, Fig. 1 shows a sketch of the frequency distribution and failure probability, where the design point represents the point on the failure surface ( $S$ $=0)$ closest to the average point in the space of normalized variables. As already mentioned, a basic quantity of reliability theory (as defined in DIN EN 1990 (2010)) is the so-called reliability $\beta$ index as a measure of an assigned probability of failure at a design point. The reliability index is usually considered to be $\beta=3.8$ for the ultimate limit state and the permanent design situation with a design life time of a building of 50 years.

As stated before, the terms safety and reliability play a crucial role in building and civil engineering, thus the safety formats used in this context (in Europa) are currently based on the semi-probabilistic safety concept as given in DIN EN 1990 (2010). Within this framework, partial safety factors are applied to actions as well as resistances, increasing the characteristic action side and reducing the characteristic resistance side. A general and schematic overview of the partial factor concept according to DIN EN 1990 (2010) is given in Fig. 2. From this graph, it can be seen directly how the partial material safety factor influences the resistance side. As already mentioned, this paper only deals with the derivation of a partial material safety factor according to EC0 for structural silicones in the building industry. It is important to note that according to the Eurocode, there is a partial safety factor $\gamma_{M}$ for a building material property and a partial safety factor $\gamma_{M}$ for a component property taking into account model uncertainties and size deviations, whereby the latter is relevant for this paper. For traditional construction materials such as concrete or steel, the partial safety 
factors in the ultimate limit states are based on theoretical considerations and experience. They essentially consist of the proportions for material scatter (represented by $\gamma_{m}$ ) and model uncertainty and scatter in geometry (represented by $R_{d}$, cf. (6)).

A limitation of this paper is that the derivation of the partial safety factor is a level I approximation according to DIN EN 1990 (2010). The Eurocode still offers the 1st Order Reliability Methods (FORM) (level II) and the fully probabilistic methods (level III). These probabilistic methods of higher levels are concerned in future papers on that topic by the authors but are omitted for reasons of brevity of this paper at this point. In contrast to the briefly described semi-probabilistic concept according to DIN EN 1990 (2010), the existing standard for dimensioning and construction of structural sealant components is regulated in ETAG 002 (2012), which is based on a global safety concept with a conservative global safety factor of $\gamma_{\text {tot }}=6$. In order to enable an improved economic and thus more sustainable design of Structural Sealant Glazing (SSG) applications, this paper provides the derivation of partial safety factors for structural silicone sealants in accordance with DIN EN 1990 (2010), following the semiprobabilistic approach. However, in order to create the link to ETAG 002 (2012), especially with regards to the ageing behaviour of silicone adhesives in façades, boundary conditions from ETAG 002 (2012) are also used in the derivation of the partial safety factor.

\section{Mathematical Basics for the Derivation of the Partial Safety Factor}

According to DIN EN 1990 (2010); Sørensen (2002), the design values of a material or product properties $X$ are determined by:

$$
X_{d}=\eta \frac{X_{k}}{\gamma_{m}}=\frac{\eta}{\gamma_{m}}\left\{m_{X}-k_{n} V_{X}\right\},
$$

where, $X_{k}$ is the characteristic strength value (5\% quantile) and $\eta$ is the mean value of a conversion factor taking into account differences between material strength in the computational model and in the actual structure as well as laboratory size effects (moisture, temperature, scale and size effects, etc.,). Usually, $\eta=$ 1 is assumed (DIN EN 1990, 2010), within this paper however, a interconnection to ETAG 002 is established via this coefficient, cf. Sec. 3.3. Furthermore, the variable $m_{X}$ represents the mean value of the material property $\mathrm{X}$ given $n$ samples, $k_{n}$ is the fractile factor for characteristic value and $V_{X}$ is the coefficient of variation for the material property $X$.

In contrast to the design values of material or product properties, the real design resistance includes additionally uncertainties in the resistance model, e.g., geometric deviations. The design resistance $R_{d}$ is then defined by:

$$
R_{d}=\frac{1}{\gamma_{R d}} R\left(\frac{\eta}{\gamma_{m}} X_{k}\right)=\frac{R_{k}}{\gamma_{M}},
$$

where, $\gamma_{M}$ is the partial material safety factor (including the uncertainty in the material property described by $\gamma_{m}$, the uncertainty in the structural model of the structural members and the geometric data defined by $\gamma_{R d}$ and $\eta$ representing the mean value of the conversion factor that takes into account volume and scale effects, the effects of moisture and temperature, etc.).

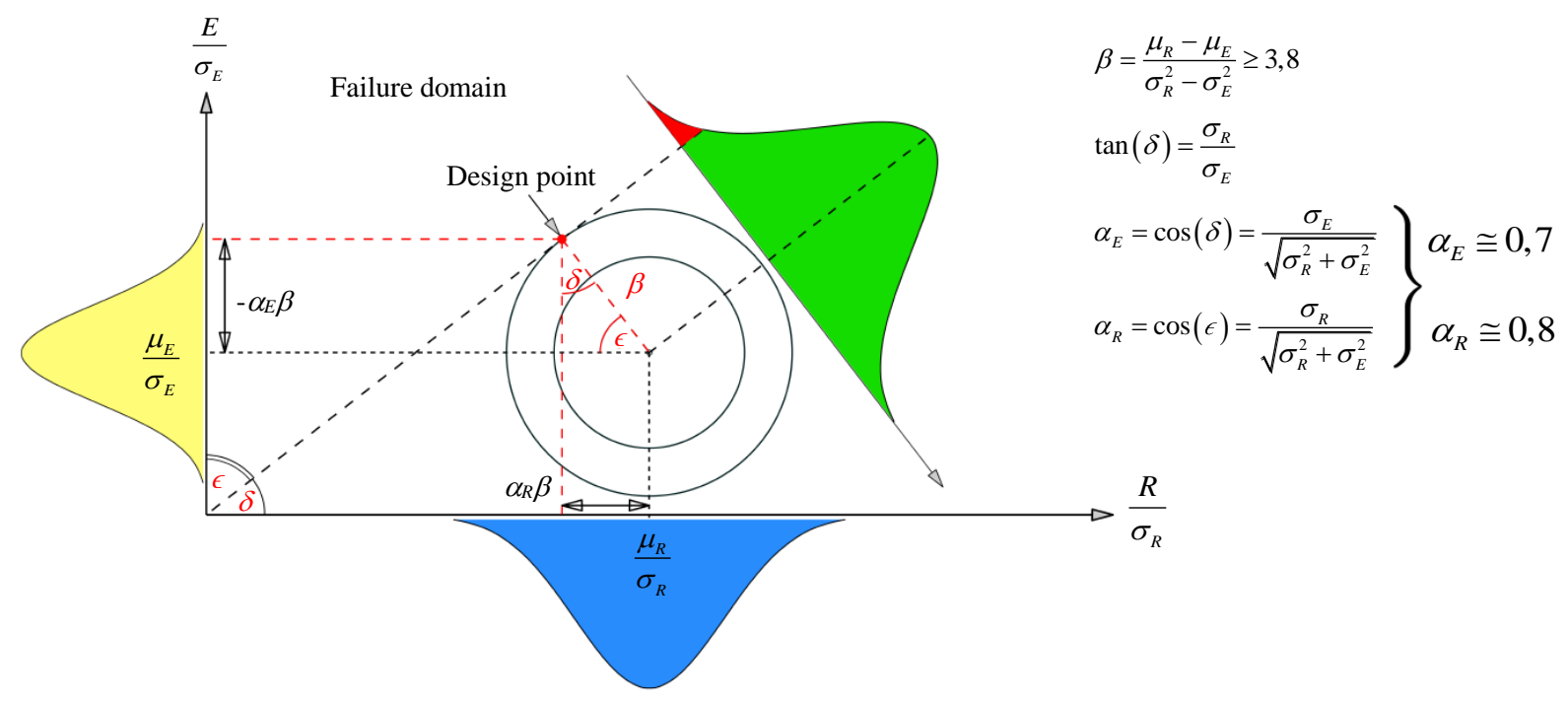

Fig. 1: Design Point (P) and reliability index $\beta$, based on DIN EN 1990 (2010), from Kroyer and Taras (2019) 


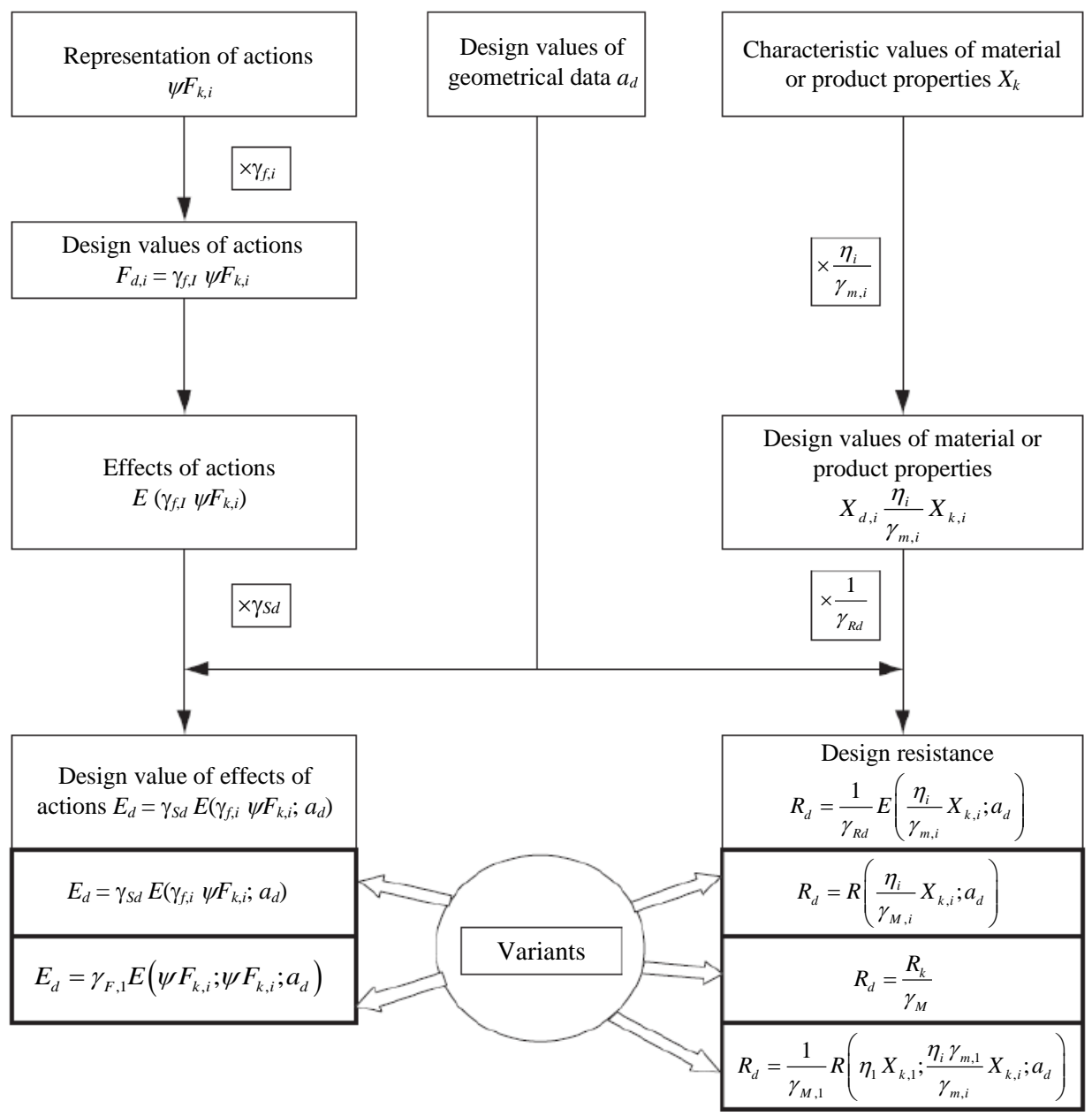

Fig. 2: General overview of the partial factor system in the Eurocodes, from Gulvanessian et al. (2012)

In order to return to the partial safety factor for a general class of building materials, it should generally be possible to calculate it according to the following equation (Sørensen, 2002), using various influencing factors:

$\gamma_{M}=\gamma_{0} \cdot \gamma_{1} \cdot \gamma_{2} \cdot \gamma_{3} \cdot \gamma_{4} \cdot \gamma_{5}$

with the factors taking into account:

- $\quad \gamma_{0}$ : Consequences of failure (safety class: low: 0.9; normal: 1.0; high: 1.1 )

- $\quad \gamma_{1}$ : Type of failure (ductile with reserve: 0.9; without reserve: 1.0 and brittle: 1.1 )

- $\quad \gamma_{2}$ : Possibility of unfavourable differences from the characteristic value of the material parameter (i.e., uncertainty related to the parameter)

- $\gamma_{3}$ : Uncertainty of the computational model (good: 0.95; normal: 1.0 ; bad: 1.1 )

- $\quad \gamma_{4}$ : Uncertainty in connection with determination of the material parameter in the structure on the basis of the controlled material parameter (large: 0.95, average: 1.0 and small: 1.1 )
- $\quad \gamma_{5}$ : Amount of control (extended: 0.95, normal: 1.0 and reduced: 1.1)

From this list, it is obvious that the calibration of a partial safety factor depends on various influencing factors, which can be determined by assumptions or exact calculation methods, which of course increases the calibration effort. It is the engineer's choice to have more insight or to put more effort into e.g., a more suitable and detailed constitutive modelling (which would potentially govern the parameters $\gamma_{2}, \gamma_{3}$ and $\gamma_{4}$ in the regions $\leq 1.0$ ) or to conduct system component analysis (potentially $\gamma_{1} \leq 1.0$ for more advanced loadpath analyses such as the plastic hinge theory etc.).

At this point, it is important to remember that the partial safety factor alone is not sufficient for the design, but that the characteristic strength value is also required for the design limit state analysis. In general, with increasing modelling and testing effort, both the partial material safety factor and the characteristic strength can be estimated with a low uncertainty. Following the simplified level I approach 
according to DIN EN 1990 (2010), whereby at this point a suitable and meaningful mechanical model is assumed, then the partial safety factor $\gamma_{\mathrm{M}}$ is computed by:

$$
\gamma_{M}=\frac{\gamma_{R d} \cdot \gamma_{m}}{\eta}=\frac{1}{\eta} \cdot \exp \left(\alpha_{R} \cdot \beta \cdot V_{R}-1.645 \cdot V_{F}\right),
$$

where, $\alpha_{R}$ represents a weighting factor for the resistance according to the level I approximation method and $\beta$ is the reliability index. The coefficient of variation is computed by:

$V_{R}=\sqrt{V_{M}^{2}+V_{G}^{2}+V_{F}^{2}}$,

where the components are defined as:

- $\quad V_{M}$ : Coefficient of variation for model uncertainty of structural silicone sealant

- $V_{\mathrm{G}}$ : Coefficient of variation for the geometry

- $V_{\mathrm{F}}$ : Coefficient of variation for the structural silicone sealant strength

When using the log-normal distribution for the material property $X$ (as it is recommended in Fischer (2001) for small sample sizes with a certain coefficient of variation), (5) can be reformulated by:

$X_{k}=\exp \left(m_{X}-k_{n} \cdot s_{X}\right)$,

which can now be used to calculate the characteristic failure strength of the analysed material, e.g., a DOWSIL 993 structural silicone. In this context, the mean value $m_{\mathrm{X}}$ of the is given by:

$m_{X}=\frac{1}{n} \sum_{i=1}^{n} \ln \left(x_{i}\right)$

and the standard deviation $s_{X}$ is given by:

$$
s_{X}=\sqrt{\frac{1}{n-1} \sum_{i=1}^{n}\left(\ln x_{i}-m_{X}\right)^{2}}
$$

By now, the computation of the characteristic value of the material property $X$ has been discussed specifically. The fractile factor $k_{n}$ is provided in Table 1 and the coefficient of variation reads:

$V_{F}=\sqrt{\exp \left(s_{X}^{2}\right)-1}$

Table 1: Values of $k_{n}$ for estimation of characteristic values (5\% fractiles), DIN EN 1990 (2010)

\begin{tabular}{llllll}
\hline $\mathrm{n}$ & 5 & 8 & 10 & 20 & $\infty$ \\
\hline Unknown $V_{X}$ & 2.33 & 2.00 & 1.92 & 1.76 & 1.64 \\
\hline
\end{tabular}

According to the formulas given above, it is now possible to calculate a partial safety factor for any material according to the level I concept of DIN EN 1990 (2010). In the remainder of this section, this approach is applied to the test data of the structural silicone DOWSIL 993.

\section{Calibration of a Partial Material Safety Factor for DOWSIL 993}

In this section, the partial material safety factor for DOWSIL 993 is calibrated based on experimental data. For this purpose, specifications from DIN EN 1990 (2010) as well as restrictions of ETAG 002 (2012) are included in order to calculate $\gamma_{M}$ for the structural silicone according to the level I method of DIN EN 1990 (2010).

Regarding (8), the weighting factor for the resistance according to the level I method is taken as $\mathrm{R}=0.8$. This factor corresponds to the specifications from DIN EN 1990 (2010) and lies on the safe side. The reliability index is usually considered as $\beta=3.8$ for the ultimate limit state, which corresponds to a permanent design situation with a target design life of the building of 50 years. For the coefficients of variation it is assumed:

- $V_{M}=0.05 / 0.10$ coefficient of variation for model uncertainty of structural silicone sealant

- $V_{\mathrm{G}}=0.05 / 0.10$ coefficient of variation for the geometry of the adhesive joint

For the subsequent computations. Here, we assume that our model inaccuracies amount to a maximum of $10 \%$ (interpreted as mean squared error). This is justified by the fact that adequate material models for structural silicones have been developed in recent years, which can reproduce the structural behaviour very accurately (Drass et al., 2018; 2019). An exact determination of the model inaccuracies is not part of this very paper, but it will be calculated exactly in a subsequent paper by the authors. As a further assumption for the calculation of the partial material safety factor, the geometric inaccuracy of the adhesive joint is also assumed to be at maximum $10 \%$. This value is almost very high if one considers that adhesive joints are applied to the components industrially in the factory under machine control.

Here again, there might be a discussion on the actual value for $V_{G}$ so that companies with a high degree of precision in the application of structural silicone could reduce this factor. To determine the influence of the uncertainties from geometry and model directly in the form of the value for $\gamma_{M}$, within this paper the evaluation for $\gamma_{M}$ takes place once for $5 \%$ and once for $10 \%$ as values for coefficients of variations $V_{M}$ and $V_{G}$.

In order to gain insight into the sensitivity of $\gamma_{M}$ in dependence of $V_{M}$ and $V_{G}$, Fig. 3 shows the results for $\gamma_{m}$ in dependence of those two sources of uncertainty. The coefficient of variation for the structural silicone sealant 
strength is calculated based on the experimental results (Table 2) and reads ca. $V_{F}=7 \%$. Within the sensitivity study, two additional values for $V_{F}$ are investigated, where it was found, that the sensitivity of $\gamma_{m}$ is more dominant for $V_{F}=1 \%$ and less for $V_{F}=10 \%$. As can be seen clearly, the value for $\gamma_{M}$ increases nonlinearly when the coefficients of variations $V_{G}$ and $V_{M}$ increase. It is also curious that the isoplanes for a constant $V_{F}$ do not run parallel to each other, but intersect. Although this behavior results from mathematics, it is to be regarded as critical from a physical point of view, since a smaller $\gamma_{M}$ is formally obtained despite a greater scattering of the material strength. Here one would have to calculate all values exactly according to experimental tests in order to check the course. According to DIN EN 1990 (2010), the coefficient of variation are therefore limited to a maximum of 0.2 in order to legitimize the procedure presented.

Since, according to (8), we have calculated $\gamma_{M}$ under the assumption, that there is no conversion factor eta needed. In order to take into account further model uncertainties and conversion issues, the conversion factor $\eta$ is now linked with requirements from ETAG 002 (2012) in order to have a reasonable assumption with regard to model uncertainty taking into account ageing effects.

Ageing phenomena typically occur in façades as a result of water, temperature, $\mathrm{UV}, \mathrm{NaCl}, \mathrm{SO}_{2}$, cleaning agent exposure effects. Accordingly to ETAG 002 (2012), these disadvantageous ageing effects are experimentally tested, in which the ratio of the aged nominal strength to the unaged strength must be greater than $75 \%$ Fig. 4. In Fig. 4, the barrier according to ETAG 002 (2012) for tensile and shear loads was drawn as a grey box according to the available experimental data. It can be seen that the mean value of the nominal strengths of the artificially aged samples is above the $75 \%$ criterion, so that the requirements of ETAG 002 (2012) are met here.

This criterion therefore provides a lower limit value which must be met experimentally in order to be able to construct an SSG façade. Assuming this lower limit value is a true barrier according to ETAG 002 (2012), which includes all harmful influences such as temperature, water and UV storage as well as salt exposure, the conversion factor $\eta$ can be determined accordingly:

$\eta=0.75$

This procedure is reasonable, creates a limit value for $\eta$ and links the ETAG 002 (2012) concept with DIN EN 1990 (2010). In the case that experimental test data under ageing are available, the conversion factor $\eta$ can be adjusted according to the results of the ageing tests, which leads to improved results in the sense of a smaller partial material safety factor $\gamma_{M}$.

Since all necessary information for the calibration and thus computation of the partial material safety factor is now available, the results for the structural silicone DOWSIL 993 are presented below. For computing the partial material safety factors for the tensile and shear strengths, two situations are differentiated: On the one hand side $\gamma_{M}$ accordingly to (8) is computed with $\eta=$ 1.0, where further ageing effects are completely disregarded. On the other hand, $\gamma_{M, E T A G}$ is additionally calculated with $\eta=0.75$ in order to account for the difference between measured strength by laboratory tests and strength of a built component considering further ageing effects. As already mentioned, $\eta$ has been chosen in such a way that the limit value defined by ETAG 002 (2012) is adhered to due to ageing.

The results for the partial material safety factor $\gamma_{M}$ and $\gamma_{\text {M,ETAG }}$ as well as characteristic failure strength $X_{k}$ for DOWSIL 993 are shown in Fig. 5 for the coefficients of variations of $5 \%$. In contrast, Fig. 6 shows the results for the partial material safety factors, where the coefficients of variations have been assumed to $10 \%$. Accordingly, we also receive slightly higher values for the partial safety factors.

Table 2: Partial material Safety Factors (PSF) $\gamma_{\mathrm{M}}$ and $\gamma_{\mathrm{M}, \mathrm{ETAG}}$ for $V_{M}=V_{\mathrm{G}}=5 / 10 \%$ as well as characteristic failure strength $X_{k}$ [MPa] for the DOWSIL 993 structural silicone

\begin{tabular}{|c|c|c|c|c|c|}
\hline & \multirow[b]{2}{*}{$\begin{array}{l}\text { Char. Failure } \\
\text { Strength } X_{k}[\mathrm{MPa}]\end{array}$} & \multicolumn{2}{|c|}{$V_{G}=V_{M}=0.05$} & \multicolumn{2}{|c|}{$V_{G}=V_{M}=0.10$} \\
\hline & & $\gamma_{M}$ & $\gamma_{\mathrm{M}, \mathrm{ETAG}}$ & $\gamma_{M}$ & $\gamma_{\mathrm{M}, \mathrm{ETAG}}$ \\
\hline Tension, unaged, $\mathrm{T}=23^{\circ} \mathrm{C}$ & 1.14 & 1.22 & 1.63 & 1.52 & 2.02 \\
\hline Tension, unaged, $\mathrm{T}=80^{\circ} \mathrm{C}$ & 0.88 & 1.23 & 1.64 & 1.53 & 2.03 \\
\hline Tension, unaged, $\mathrm{T}=-20^{\circ} \mathrm{C}$ & 1.52 & 1.21 & 1.61 & 1.50 & 1.99 \\
\hline Shear, unaged, $\mathrm{T}=23^{\circ} \mathrm{C}$ & 0.77 & 1.22 & 1.63 & 1.51 & 2.01 \\
\hline Shear, unaged, $\mathrm{T}=80^{\circ} \mathrm{C}$ & 0.61 & 1.23 & 1.64 & 1.53 & 2.03 \\
\hline Tension, aged-water, $\mathrm{T}=23^{\circ} \mathrm{C}$ & 1.08 & 1.24 & 1.64 & 1.53 & 2.04 \\
\hline Tension, aged- $\mathrm{NaCl}, \mathrm{T}=23^{\circ} \mathrm{C}$ & 1.24 & 1.23 & 1.64 & 1.52 & 2.03 \\
\hline Tension, aged-SO $\mathrm{S}_{2}, \mathrm{~T}=23^{\circ} \mathrm{C}$ & 1.17 & 1.23 & 1.64 & 1.53 & 2.04 \\
\hline Tension, aged-detergent, $\mathrm{T}=23^{\circ} \mathrm{C}$ & 1.06 & 1.23 & 1.63 & 1.52 & 2.02 \\
\hline Tension, unlabelled & 1.11 & 1.25 & 1.66 & 1.54 & 2.05 \\
\hline Shear, unlabelled & 0.78 & 1.22 & 1.63 & 1.50 & 1.99 \\
\hline
\end{tabular}




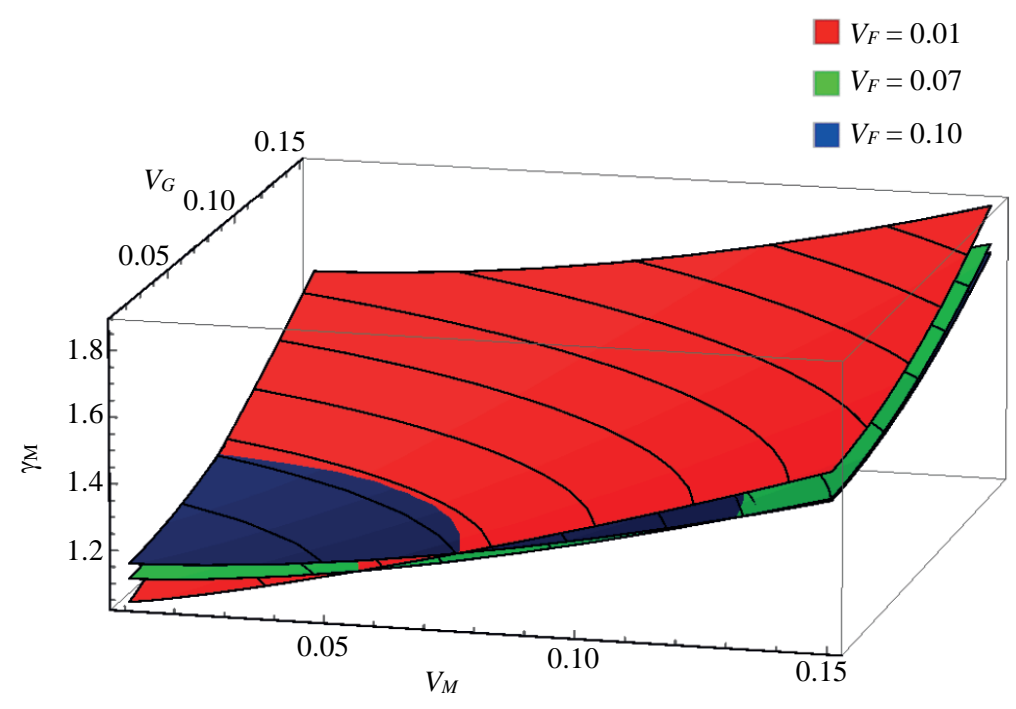

Fig. 3: Results of the parametric study for the sensitivity of $\gamma_{M}$ w.r.t. the coefficients of variation for geometry and model uncertainty $V_{M}$ and $V_{G}$

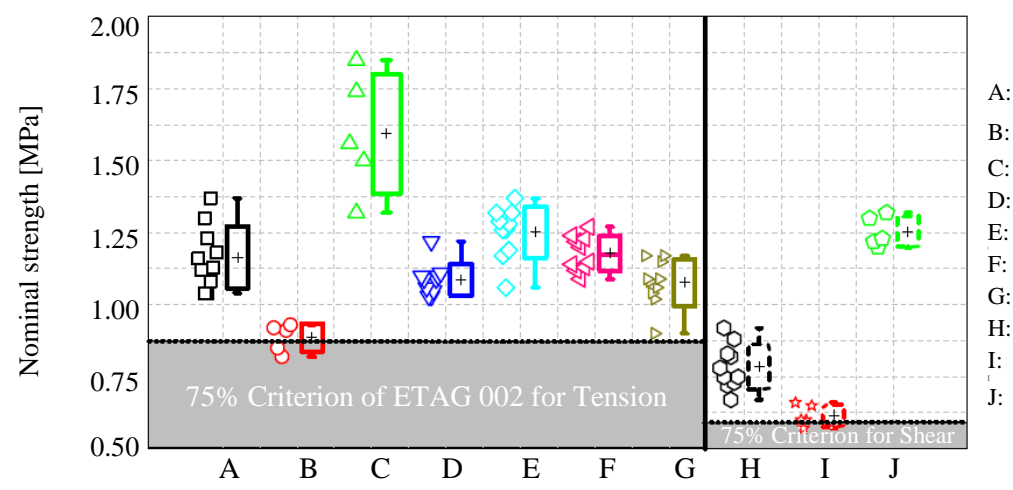

\section{Tem/ageing}

$\square \mathrm{T}=+23^{\circ} \mathrm{C} /$ None

$\mathrm{T}=+80^{\circ} \mathrm{C} / \mathrm{None}$

$\mathrm{T}=-20^{\circ} \mathrm{C} /$ None

$\mathrm{T}=+23^{\circ} \mathrm{C} /$ Water

$\mathrm{T}=+23^{\circ} \mathrm{C} / \mathrm{NaCl}$

$\mathrm{T}=+23^{\circ} \mathrm{C} / \mathrm{SO}_{2}$

$\mathrm{T}=+23^{\circ} \mathrm{C} /$ Cleaning agent

$\mathrm{S}=+23^{\circ} \mathrm{C} / \mathrm{None}$

$\mathrm{S}=+80^{\circ} \mathrm{C} / \mathrm{None}$

$\mathrm{S}=-20^{\circ} \mathrm{C} /$ None

I $5 \%$ $95 \%$

Fig. 4: Box plot of nominal failure strengths of DOWSIL 993 under Tension (T) and Shear (S) loading

2,00
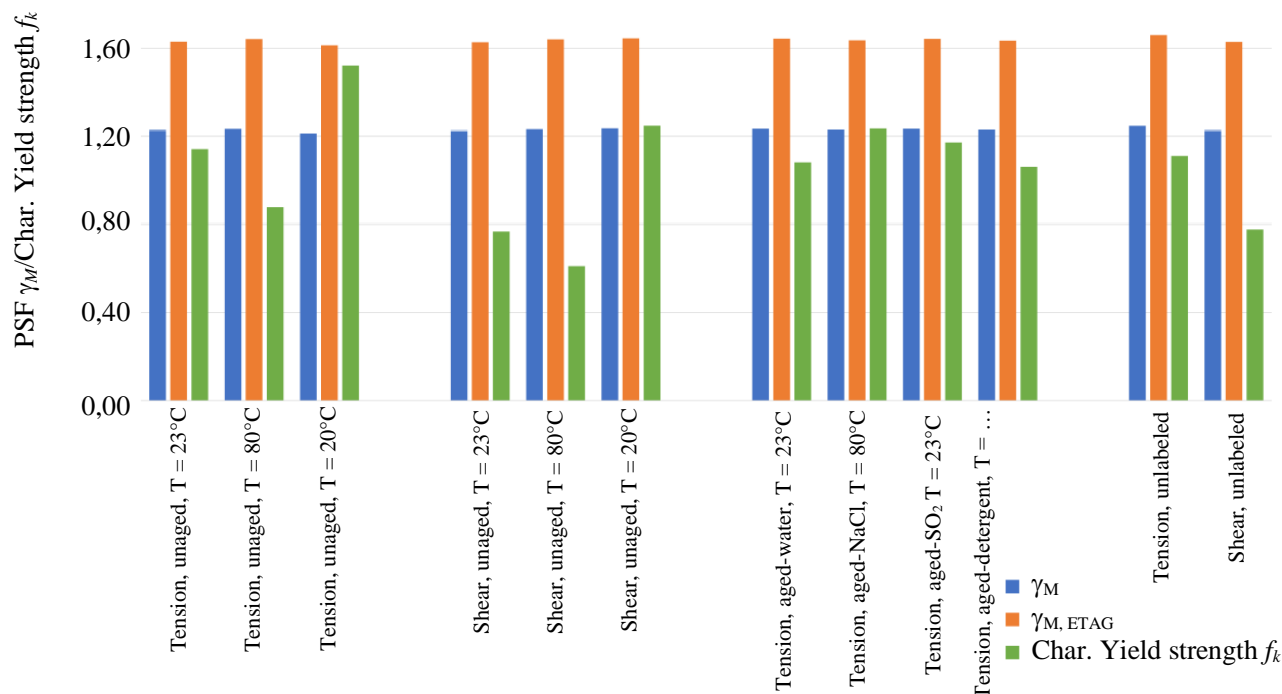

Fig. 5: Partial Material Safety Factors (PSF) $\gamma_{M}$ and $\gamma_{\mathrm{M}, \mathrm{ETAG}}$ for $V_{M}=V_{\mathrm{G}}=5 \%$ as well as characteristic failure strength $X_{\mathrm{k}}[\mathrm{MPa}]$ for the DOWSIL 993 structural silicone 


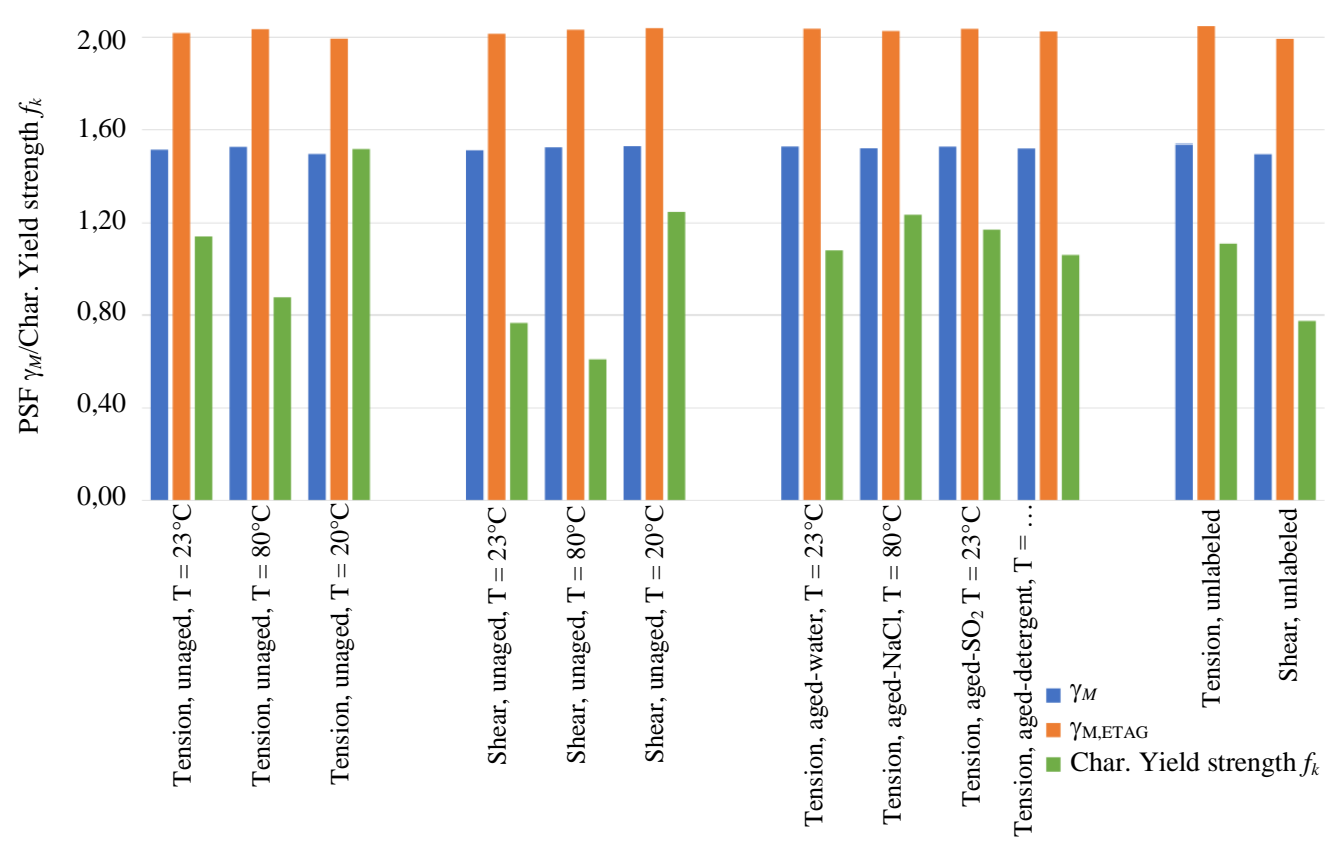

Fig. 6: Partial Material Safety Factors (PSF) $\gamma_{M}$ and $\gamma_{M}$,ETAG for $V_{M}=V_{G}=10 \%$ as well as characteristic failure strength $X_{k}$ [MPa] for the DOWSIL 993 structural silicone

The difference in $\gamma_{M}$ and $\gamma_{\mathrm{M}, \mathrm{ETAG}}$ is, that for $\gamma_{M}$ the value for $\eta=1$ and for $\gamma_{M}$ the value for $\eta=0.75$ is used. The graphs show the safety factors for different design situations (temperatures and ageing conditions) as well as an 'overall' safety factor, for which it has been assumed that all performed tests are considered without a labelling for the specific testing/ageing protocol. This means, that a partial material safety factor with an associated characteristic yield strength is elaborated for the 'general design situation', which does not specifically consider temperature and ageing but rather computes the statistical properties over all test results. It is interesting to see, that the partial material safety factor $\gamma_{M}$ is almost insensitive of the applied degradation and loading scenario whereas the characteristic yield strengths are. To also be able to read the absolute values for the partial material safety factors, these are summarized in Table 2.

\section{Discussion of the Determined Partial Material Safety Factor for Structural Silicone}

Having obtained numerical values for the partial material safety factor with associated characteristic values for the DOWSIL 993, this section discusses the underlying assumptions more in detail.

Following Table 2, it is interesting to note that the partial material safety factor, including the consideration of ageing effects, is $\leq 2.0$. This result clearly shows that the global safety factor according to ETAG 002 (2012) is very much on the safe side. In order to create direct comparability between the partial material safety factor according to EC0 and the global safety factor $\gamma_{\text {tot }}$ according to ETAG 002 (2012), it is assumed when converting $\gamma_{M}$ into a global safety factor that only live loads affect the component. As a result, $\gamma_{M}$ is now multiplied by the partial safety factor on the action side of 1.5 , resulting in a conservative global safety factor of:

$\gamma_{t o t, 5 \%}^{*}=\gamma_{M} \cdot \gamma_{E d}=1.64 \cdot 1.5=2.46 \ll 6.0$.

and:

$\gamma_{t o t, 10 \%}^{*}=\gamma_{M} \cdot \gamma_{E d}=2.02 \cdot 1.5=3.03 \ll 6.0$.

If one compares this with the global safety factor according to ETAG 002 (2012), a reduction of more than $200 \%$ results, despite consideration of damaging influences. However, it should be mentioned again that this value is based on the assumption that the model and geometry uncertainties are within 5-10\%. Therefore, the premise for the application of the proposed concept is to apply suitable material models and failure criteria for the investigated structural silicone in order to enable the smallest possible uncertainty on the material side. The uncertainty for the geometry of the adhesive joint can be individually adapted. Although an uncertainty of $5-10 \%$ was assumed here, it should be noted that the application of adhesive joints takes place mechanically in the factory, so that this assumption is justified.

Furthermore, regarding Sec. 3.1, a safety margin equation $g(E, R)$ of demand $E$ and resistance $R$ has to be provided. This relationship $g(E, R)$ was not further 
specified within the context of this paper, as the semiprobabilistic deduction of a partial material safety factor can be done accordingly to DIN EN 1990 (2010) for a conservative choice of the corresponding parameters, cf. Sec. 3.3. However, the following points allow for more detailed and precise computation of the partial material safety factor in future research:

- 'Realistic' ageing protocols (deduction of load combination factors)

- Fatigue

- Viscoelasticity

- Different performance/limit state functions $g(E, R)$

- Multiple failure modes (distortional and/or dilatation) of the sealant

- $\quad$ Failure modes of the sealing application and the sealed system (series and/or parallel system behaviour)

The actual numerical values for the resistance measure can be sensitive to the formulation of failure, i.e., the choice of the parameters, how the ultimate failure of the analysed material is measured. Within this paper, tensile and shear yield strengths (engineering measure) were used to formulate the failure event. Thus, the computed partial material safety factors are strictly speaking just applicable to the structural silicone adhesive DOWSIL 993, when the design of the adhesive joint is formulated and computed in an ETAG 002 (2012) framework. Consequently, the real material behaviour and failure description of the sealant is not considered within the limit state function.

As many rubber-like materials are nearly incompressible until fracture due to incompressibility, failure may not only occur due to distortion but also because of dilatation. The computed partial material safety factors as presented within this paper just cover dilatational failure. Further recommendations on how to deal with different mechanical demands or arbitrary silicone adhesive joints are future work and not considered within this paper. Nevertheless, the presented method is generally valid, so that it can be extended with respect to an exact material modelling and failure description. This enables the engineer to safely calculate any adhesive joints, such as point holders, 3-flank and non-ETAG adhesive joints.

Finally, this paper assumes that the ETAG ageing protocol adequately represents the realistic structural deterioration of silicone sealants during their lifetime. The statistical properties for the structural silicone under the relevant load conditions of temperature and concentration of the chemicals exist to date only according to the specifications of ETAG. However, it is questionable whether this test protocol really reflects the ageing behaviour of structural silicones. This was discussed by Fumihiko et al. (2019), during whose studies a 31-yearold SSG façade was dismantled and subsequently the load-bearing behaviour of the silicone was tested after a real ageing of 31 years. The results show amazingly that the strength of the silicone has increased with ageing, so that a discussion has to be started to what extent the ETAG ageing concept is representative at all.

Taking into account the points raised within this subsection, a more precise level II or III method for the determination of a partial material safety factor for structural sealant silicones is desirable but a complex and complicated task and is subject to future research of the authors.

\section{Discussion}

The content of this paper is devoted to the elaboration of material yield strength statistics of the DOWSIL 993 structural silicone and the determination of appropriate material partial safety factors for structural (re)assessment. The paper comprises pertinent statistics for material strength properties in the era since about 2010 until today. For the purpose of determination of adequate partial safety factors, a semi-probabilistic approach in accordance with Eurocode 1990 was proposed. In order to illustrate the procedure, the methodical outline concludes with an exemplary probabilistic evaluation of a specific limit state data for the structural silicone adhesive DOWSIL 993. The methodology and the documented silicone sealant material statistics are certainly not limited to this case of application but could be applied to other products in the market.

The partial material safety factors determined here only apply in connection with the associated characteristic strengths. It should be noted that the ETAG 002 test protocols only maps or analyses isochoric failure. Effects from volumetric damage such as cavitation failures are not represented by the ETAG 002, since there are geometrical specifications regarding the geometry of the bonded joints according to ETAG 002. Further hints on that will be treated in part II of this paper.

If within the framework of the semi-probabilistic calibration of partial material safety factors other material properties $X$ than the log-normally distributed engineer yield stresses are used for the limit state function, other partial safety factors may be obtained as a result (this will be the subject of future publications by the authors), since deviating coefficients of variation for the material property $X$ as well as the coefficient of variation of the model uncertainty $V_{M}$ are potentially obtained. Furthermore, the uncertainty for the geometry of the bonded joint can be adapted individually. The value for the coefficient of variation of the geometry $V_{G}$ 
as chosen within the scope of this article with $10 \%$ is interpreted as rather conservative, since load-bearing adhesive joints are usually industrially manufactured products and thus have a high accuracy.

Finally, the calibration of the material partial safety factors in this publication assumes that the ETAG ageing protocols adequately represent realistic thermomechanical-chemical stresses of silicones in SSG facades during their service life. The statistical properties of DOWSIL 993 under the respective ageing conditions of temperature and concentration of the chemicals exist so far only according to the specifications of ETAG 002 and have been taken into account in this article. However, due to contrary experimental findings it is questionable whether the ETAG test protocol really reflects the ageing behaviour of SSG silicones (as discussed above) in a real-world application.

\section{Conclusion}

The values determined within this paper show that the material partial safety factors (even taking into account temperature and ageing and laboratory effects) are approx. 2 and thus a global partial safety factor of 3 can be justified, which in future will lead to a more appropriate, economic and sustainable design of silicone joints.

Taking into account the points mentioned in the last section, a more precise method of level II or III acc. To DIN EN 1990 (2010) is desirable for calibration of a partial material safety factor for structural silicone sealants with a more precise representation of the mechanical load-bearing behaviour in the sense of a more adequate limit state function.

\section{Acknowledgement}

We would like to express our greatest gratitude to Dr. Ing. Martien Teich from Seele $\mathrm{GmbH}$ as well as to M.Sc. Robert Kroyer jun. (Bundeswehr University Munich) for providing the experimental data, a figure and many fruitful discussions on various aspects of this work.

\section{Author's Contributions}

Michael Drass: Checked numerical simulations, coordinated and conducted the data-analysis and derivation of results and contributed to the writing of the manuscript and gave final approval for this article.

Michael A. Kraus: Conducted numerical experiments and data-analysis, double-checked the derivation of the results ant the data-analysis and contributed to the writing of the manuscript and gave final approval for this article.

\section{Ethics}

This article is original and contains unpublished material. The corresponding author confirms that all of the other authors have read and approved the manuscript and no ethical issues involved.

\section{References}

ASTM C1401, 2002. Standard guide for structural sealant glazing.

Bedon, C. and M. Santarsiero, 2018 Transparency in structural glass systems via mechanical, adhesive and laminated connections-existing research and developments. Adv. Eng. Mater., 20: 1700-815. DOI: 10.1002/adem.201700815

DIBt Concept, 2012. Vorschlag: Zeitstandnachweis für geklebte lastabtragende verbindungen.

DIN EN, 1990, 2010. Eurocode: Grundlagen der tragwerksplanung. Deutsche Fassung EN 1990: 2002+ Al: 2005+ A1: 2005/AC: 2010.

Drass, M., 2019. Constitutive modelling and failure prediction of silicone adhesives in facade design. PhD Thesis, Technische, Universität Darmstadt.

Drass, M., G. Schwind, J. Schneider and S. Kolling, 2018. Adhesive connections in glass structures-part ii: Material parameter identification on thin structural silicone. Glass Structures Eng., 3: 55-74. DOI: 10.1007/s40940-017-0048-3

Drass, M., P.A. Du Bois, J. Schneider and S. Kolling, 2019. Pseudoelastic cavitation model-part i: Finite element analyses on thin silicone adhesives in façades. Glass Structures Eng.

ETAG 002, 2012. Guideline for European technical approval for structural sealantglazing kits.

Fischer, L., 2001. Das neue sicherheitskonzept im Bauwesen: Ein Leitfaden für Bauingenieure, Architekten und Studenten, Spezial, Ernst. Bautechnik.

Fumihiko, C., M. Takashi and M. Hideyuki, 2019. Structural silicone performance after 31 years in service in japan. Proceedings of the Conference: All Eyes on Smarter Glass, (ESG' 19), Glass Performance Days.

Gulvanessian, H., J.A. Calgaro and M. Holicky, 2012. Designers' guide to eurocode: Basis of structural design.

Kroyer, R. and A. Taras, 2019. Material strength statistics and reliability aspects for the reassessment of end-of-service-life steel bridges. Proceedings of the International Colloquium on Stability and Ductility of Steel Structures, Sept. 11-13, Prague, Czech Republic.

Maniatis, I. and G. Siebert, 2016. A new design approach for structural bonded silicone joints. Proceedings of the GlassCon Global USA, (IGT' 16), Innovation in Glass Technology, pp: 159-164. 
Maniatis, I., G. Siebert and G. Nehring, 2015. Dimensionierung von klebefugen aus silikon mit beliebiger geometrie. Stahlbau, 84: 293-302.

Santarsiero, M. and C. Louter, 2019. Metal-to-glass bond strength of structural PVB. Proceedings of the GPD Glass Performance Days, (GPD’ 19).

Santarsiero, M., 2015. Laminated connections for structural glass applications. PhD Thesis, Swiss Federal Institute of Technology.
Sørensen, J.D., 2002. Calibration of partial safety factors in Danish structural codes. Proceedings of the JCSS Workshop on Reliability Based Code Calibration, Mar. 21-22, Zurich.

Staudt, Y., C. Odenbreit and J. Schneider, 2018. Failure behaviour of silicone adhesive in bonded connections with simple geometry. Int. J. Adhes. Adhesives, 82: 126-138.

DOI: 10.1016/j.ijadhadh.2017.12.015 